\section{Fontes de obtenção de medicamentos por pacientes diagnosticados com doenças crônicas, usuários do Sistema Único de Saúde}

\author{
Sources of medicines for chronic patients using \\ the Brazilian Unified National Health System
}

\author{
Samara Ramalho Matta 1,2 \\ Andréa Dâmaso Bertoldi 3 \\ Isabel Cristina Martins Emmerick 2 \\ Andréia Turmina Fontanella 4 \\ Karen Sarmento Costa 5 \\ Vera Lucia Luiza 2 \\ Grupo PNAUM 6
}

doi: 10.1590/0102-311X00073817

\title{
Resumo
}

Este artigo teve como objetivo identificar as fontes de obtenção de medicamentos utilizadas por usuários do Sistema Único de Saúde (SUS) com relato de doenças crônicas, caracterizando tal população quanto a variáveis demográficas, socioeconômicas e de saúde nas diferentes regiões do Brasil. Foram analisados dados da Pesquisa Nacional sobre o Acesso, Utilização e Promoção do Uso Racional de Medicamentos no Brasil (PNAUM), um estudo transversal de base populacional. A variável dependente foi a "fonte de obtenção de medicamentos" e foram analisadas associações com variáveis demográficas, socioeconômicas e com o estado de saúde. Os usuários que obtiveram cuidado e medicamentos unicamente no SUS foram aqui designados como SUS-exclusivos. Cerca de 39\% das pessoas obtiveram seus medicamentos exclusivamente em farmácias do SUS e 28,5\% recorreram a outras fontes. Foram encontrados 42,9\%, 41,8\%, 40,2\%, 31\%, usuários SUS-exclusivos, respectivamente, no Sudeste, Sul, Norte, e Nordeste. Os usuários do SUS têm como fonte primária de medicamentos o próprio SUS, entretanto há uma participação relevante de outras fontes de obtenção, indicando problemas no acesso dentro do SUS. No Sul e Sudeste, a população busca menos a farmácia privada do que no Nordeste e Norte do país.

Acesso aos Serviços de Saúde; Sistema Único de Saúde; Doença Crônica; Preparações Farmacêuticas

\author{
Correspondência \\ S. R. Matta \\ Instituto Federal de Educação, Ciência e Tecnologia do \\ Rio de Janeiro. \\ Rua Prof. Carlos Wenceslau 343, Rio de Janeiro, RJ \\ 21710-240, Brasil. \\ samara.matta2@gmail.com \\ 1 Instituto Federal de Educação, Ciência e Tecnologia do Rio de \\ Janeiro, Rio de Janeiro, Brasil. \\ 2 Escola Nacional de Saúde Pública Sergio Arouca, Fundação \\ Oswaldo Cruz, Rio de Janeiro, Brasil. \\ 3 Programa de Pós-graduação em Epidemiologia, Universidade \\ Federal de Pelotas, Pelotas, Brasil. \\ 4 Programa de Pós-graduação em Epidemiologia, Universidade \\ Federal do Rio Grande do Sul, Porto Alegre, Brasil. \\ 5 Núcleo de Estudos de Políticas Públicas, Universidade Estadual \\ de Campinas, Campinas, Brasil. \\ 6 Outros membros listados ao fim do artigo.
}




\section{Introdução}

Estudos de carga de doença realizados no Brasil têm acompanhado a transição epidemiológica e demonstrado a predominância das doenças crônicas não transmissíveis (DCNT) - doenças cardiovasculares, doenças respiratórias crônicas, diabetes, câncer e outras - embora as doenças transmissíveis ainda desempenhem um papel importante. Em 2007, aproximadamente $72 \%$ de todas as mortes no Brasil foram atribuídas às DCNT, 10\% a doenças infecciosas ou parasitárias e 5\% a distúrbios de saúde materno-infantil 1.

No Brasil, a análise do perfil das dez DCNT investigadas na Pesquisa Nacional de Saúde de 2013 (PNS 2013) identificou como mais prevalentes a hipertensão arterial (21,4\%), depressão (7,6\%), artrite $(6,4 \%)$ e diabetes mellitus $(6,2 \%)^{2}$.

O uso adequado de medicamentos como parte do cuidado integral possibilita o controle de doenças, redução de morbimortalidade e melhoria da qualidade de vida dos usuários portadores de diversas condições de saúde. Assim, a garantia de acesso aos medicamentos se torna uma estratégia fundamental às políticas voltadas aos portadores de DCNT.

O estudo de Oliveira et al. 3, realizado com os dados da Pesquisa Nacional sobre Acesso, Utilização e Promoção do Uso Racional de Medicamentos (PNAUM) coletados em 2013-2014 no Brasil mostrou alta prevalência de acesso total a medicamentos para DCNT em adultos e idosos (94,3\%).

Medicamentos para as doenças crônicas podem ser adquiridos gratuitamente nas farmácias do Sistema Único de Saúde (SUS) e no Programa Farmácia Popular do Brasil (PFPB). No caso do primeiro mecanismo, todos os medicamentos são gratuitos e destinados às DCNT mais prevalentes e, no segundo, o elenco de medicamentos é reduzido e ofertado mediante copagamento, sendo apenas alguns deles gratuitos, caso que inclui o tratamento da hipertensão, do diabetes e asma. Outra forma de acesso a medicamentos para doenças crônicas no Brasil é a obtenção mediante pagamento no setor privado.

O acesso a medicamentos para as DCNT e a outros medicamentos por intermédio da rede pública depende, entre outros fatores, de um financiamento adequado. Além disso, as dificuldades para o acesso também podem estar relacionadas à gestão dos serviços de saúde e da assistência farmacêutica em particular. De fato, o bom desempenho de atividades dos serviços farmacêuticos mostrou-se associado com a disponibilidade de medicamentos de qualidade para a população 4 .

A disponibilidade de medicamentos mediante copagamento pelos usuários foi uma ação implantada pelo governo brasileiro em 2004 5. Inicialmente, o PFPB foi instituído por uma rede própria de farmácias estatais. Atualmente, também funciona com a modalidade Aqui Tem Farmácia Popular, que resultou da parceria com o setor privado farmacêutico varejista. Em 2011, novas mudanças ocorreram, com a isenção de copagamento de um conjunto de medicamentos específicos, com a modalidade do programa chamada Saúde Não Tem Preço (SNTP) 5,6.

A despeito da ausência de lei nacional específica, o mais comum é que os estabelecimentos públicos de dispensação, em especial na atenção primária, atendam apenas às receitas geradas em sua própria rede municipal. O PFPB, por outro lado, é universal, atendendo a receitas de qualquer origem, no entanto o elenco disponível, sobretudo na modalidade Aqui Tem Farmácia Popular, com maior capilaridade no país que a modalidade de rede própria 6, é mais restrito que o elenco da assistência farmacêutica básica 5 .

É razoável supor a existência de diferenças nas proporções de uso da farmácia popular, farmácia do SUS e farmácia privada pelos usuários do SUS com doenças crônicas entre as regiões do país, já que existem desigualdades regionais quanto à rede de farmácias conveniadas ao PFPB e à situação socioeconômica da população. Ampla revisão sistemática sobre copagamento para medicamentos não identificou relato de experiências com a superposição de público-alvo de mecanismos coexistentes, como ocorre no Brasil 7.

Portanto, o objetivo deste artigo é identificar as fontes de obtenção de medicamentos utilizadas pelos usuários do SUS com relato de doenças crônicas, caracterizando essa população quanto a variáveis demográficas, socioeconômicas e de saúde nas diferentes regiões do país. 


\section{Métodos}

Foram analisados dados provenientes do inquérito domiciliar na PNAUM, um estudo transversal de base populacional. Foram realizadas entrevistas face a face em áreas urbanas, no período entre setembro de 2013 e fevereiro de 20148.

A seleção da amostra foi feita em três estágios: município (unidade primária), setor censitário e domicílio. A seleção de indivíduos dentro dos domicílios se baseou na proporção esperada de cada grupo de idade e sexo para compor a amostra final. Pesos amostrais foram calculados para o indivíduo e pesos de pós-estratificação foram utilizados para reduzir o vício decorrente da taxa de resposta 8.

A complexidade do processo amostral garantiu a representatividade nacional e para as cinco regiões do Brasil estratificadas por sexo e grupos etários em oito domínios demográficos 8 .

O questionário do inquérito domiciliar da PNAUM utilizado para adultos continha 11 blocos temáticos (1- informações do entrevistado; 2- doenças crônicas; 3- serviços de saúde; 4- doenças e eventos agudos tratados com medicamentos; 5 - contraceptivos; 6- serviços de farmácia; 7 - comportamentos que podem afetar o uso de medicamentos; 8- bulas e embalagens; 9- estilo de vida; 10- plano de saúde; e 11- informações sobre o domicílio e pessoa de referência) e duas fichas de detalhamento dos medicamentos (ficha de medicamentos para doenças crônicas e ficha de medicamentos para doenças e eventos agudos) 8 .

Para o presente estudo, foram incluídos indivíduos de 20 anos ou mais que referiram diagnóstico médico de pelo menos uma doença crônica, com indicação de tratamento medicamentoso e que declarou tratar essa(s) doença(s) com médico do SUS. Essa população foi selecionada pelas respostas às perguntas dos blocos $1,2 \mathrm{e} 3$ do questionário da PNAUM.

A variável dependente foi a "fonte de obtenção de medicamentos". A pergunta "Onde o(a) Sr.(a) obteve este remédio?" da ficha de medicamentos para doenças crônicas do Bloco 2 do questionário foi feita para cada medicamento que o entrevistado disse que tomava para o tratamento da(s) doença(s) citada(s). Para cada uma das principais fontes de obtenção (SUS, farmácia popular e farmácia privada), criou-se uma variável dicotômica que indicava se o medicamento fora obtido nessa fonte 8 .

A combinação de fontes de obtenção de medicamentos utilizada por cada indivíduo resultou em uma variável dependente com sete categorias (exclusivamente SUS; exclusivamente farmácia popular; exclusivamente farmácia privada; SUS e farmácia popular; SUS e farmácia privada; farmácia popular e farmácia privada; SUS, farmácia popular e farmácia privada) que foram reagrupadas para fins de análise:

- SUS-exclusivos: refere-se à categoria exclusivamente SUS da variável de fonte de obtenção;

- Usuários da farmácia popular: agrupamento da categoria exclusivamente farmácia popular com a categoria SUS e farmácia popular;

- Usuários da farmácia privada: agrupamento da categoria exclusivamente farmácia privada com a categoria farmácia popular e farmácia privada; $\mathrm{e}$

- Usuários de todas as fontes: agrupamento da categoria SUS e farmácia privada com a categoria SUS, farmácia popular e farmácia privada.

As variáveis independentes analisadas foram:

- Demográficas: região geográfica de residência (Norte, Nordeste, Sul, Sudeste, Centro-oeste); situação conjugal (com companheiro, sem companheiro); cor da pele autorreferida (branca, não branca); idade (20-39 anos, 40-59 anos, 60 anos ou mais); sexo (masculino, feminino);

- Socioeconômicas: escolaridade em anos de estudo (não estudou, 1-8, 9-11, 12 ou mais); classificação econômica de acordo com o Critério de Classificação Econômica Brasil 20139 (A, B, C, D ou E); filiação a plano de saúde ou convênio médico; e

- Estado de saúde: número de doenças crônicas (1, 2, 3, 4 e mais); número de medicamentos crônicos $(0,1,2,3-4,5$ ou mais); autopercepção da saúde (muito bom/bom, regular, muito ruim/ruim); diagnóstico médico autorreferido das seguintes doenças crônicas: hipertensão arterial, diabetes, doença cardíaca, hipercolesterolemia, acidente vascular cerebral, doença respiratória pulmonar crônica, doença articular crônica, depressão ou outra doença crônica com mais de seis meses de duração.

Após tratamento de algumas variáveis objetivando corrigir inconsistências e recodificar informações perdidas, foram realizadas análises uni e bivariadas. Foi utilizado o programa Stata, versão 12.0 (StataCorp LP, College Station, Estados Unidos) com aplicação do comando svy, que considera 
os aspectos de uma amostragem complexa. A associação entre a variável dependente e as variáveis independentes foi verificada pelo teste qui-quadrado de Pearson, com nível de significância de 5\%.

A PNAUM foi aprovada pela Comissão Nacional de Ética em Pesquisa (protocolo no 18947013.6.0000.0008) e pelo Comitê de Ética em Pesquisa da Universidade Federal do Rio Grande do Sul (protocolo no 19997). As entrevistas foram realizadas após assinatura de termo de consentimento livre e esclarecido por parte do entrevistado ou seu responsável legal no caso de incapazes de responder a seu próprio questionário.

\section{Resultados}

A amostra total da PNAUM incluiu 41.433 indivíduos. Este estudo abordou 6.889 indivíduos de 20 anos ou mais que relataram pelo menos uma doença crônica, que tinham indicação de uso de medicamentos e que se tratavam com médico do SUS.

A maior parte da população entrevistada foi da Região Sudeste (52\%); a menor da Norte (4,4\%). A maioria foi constituída de indivíduos que possuíam companheiro $(60,3 \%)$, não brancos $(54,8 \%)$, com mais de 40 anos de idade (84,9\%) e do sexo feminino (65,7\%). Os idosos (60 anos ou mais) representaram 40,4\% da população. Quanto à escolaridade, 15,4\% dos indivíduos não estudaram e a maioria $(43,3 \%)$ estudou de 1 a 8 anos. Mais da metade da população em estudo (58,2\%) pertencia à classe econômica C, e 89,3\% não possuíam plano de saúde ou convênio médico (Tabela 1).

\section{Tabela 1}

Distribuição de adultos e idosos (20 anos ou mais) usuários do Sistema Únido de Saúde (SUS) com relato de doença crônica não transmissível (DCNT) e com indicação de tratamento medicamentoso segundo variáveis demográficas, socioeconômicas e de saúde. Pesquisa Nacional sobre o Acesso, Utilização e Promoção do Uso Racional de Medicamentos no Brasil (PNAUM), Brasil, 2014 ( $N$ = 6.889).

\begin{tabular}{|c|c|c|}
\hline \multirow[t]{2}{*}{ Variável } & \multicolumn{2}{|c|}{ Amostra * } \\
\hline & $\%$ & IC95\% \\
\hline \multicolumn{3}{|l|}{ Região } \\
\hline Norte & 4,4 & $3,2-5,8$ \\
\hline Nordeste & 20,7 & $16,5-25,5$ \\
\hline Sudeste & 52,0 & $45,6-58,4$ \\
\hline Sul & 15,5 & $12,2-19,4$ \\
\hline Centro-oeste & 7,5 & $5,7-9,7$ \\
\hline \multicolumn{3}{|l|}{ Situação conjugal } \\
\hline Com companheiro & 60,3 & $58,2-62,3$ \\
\hline Sem companheiro & 39,7 & $37,7-41,8$ \\
\hline \multicolumn{3}{|l|}{ Cor da pele } \\
\hline Branca & 45,2 & $42,4-48,2$ \\
\hline Não branca & 54,8 & $51,8-57,6$ \\
\hline \multicolumn{3}{|l|}{ Idade (anos completos) } \\
\hline $20-39$ & 15,1 & $13,3-17,2$ \\
\hline $40-59$ & 44,5 & $42,5-46,5$ \\
\hline 60 ou mais & 40,4 & $38,4-42,3$ \\
\hline \multicolumn{3}{|l|}{ Sexo } \\
\hline Masculino & 34,3 & $32,73-36,0$ \\
\hline Feminino & 65,7 & $64,0-67,3$ \\
\hline
\end{tabular}

(continua) 
Tabela 1 (continuação)

\begin{tabular}{|c|c|c|}
\hline \multirow[t]{2}{*}{ Variável } & \multicolumn{2}{|c|}{ Amostra * } \\
\hline & $\%$ & IC95\% \\
\hline \multicolumn{3}{|l|}{ Escolaridade (anos de estudo) } \\
\hline Não estudou & 15,4 & $13,8-17,0$ \\
\hline $1-8$ & 43,3 & $40,9-45,8$ \\
\hline $9-11$ & 31,1 & $29,0-33,2$ \\
\hline 12 ou mais & 10,2 & $8,9-11,8$ \\
\hline \multicolumn{3}{|l|}{ Classificação econômica ** } \\
\hline $\mathrm{A} / \mathrm{B}$ & 17,9 & $16-20,0$ \\
\hline C & 58,2 & $56,3-60,0$ \\
\hline $\mathrm{D} / \mathrm{E}$ & 23,9 & $21,7-26,2$ \\
\hline \multicolumn{3}{|l|}{ Plano de saúde ou convênio médico } \\
\hline Sim & 10,7 & $9,2-12,2$ \\
\hline Não & 89,3 & $87,7-90,7$ \\
\hline \multicolumn{3}{|l|}{ Autopercepção da saúde } \\
\hline Muito bom/Bom & 47,4 & $44,9-49,9$ \\
\hline Regular & 43,2 & $41,0-45,4$ \\
\hline Muito ruim/Ruim & 9,4 & $8,4-10,6$ \\
\hline Hipertensão arterial & 69,7 & $67,9-71,4$ \\
\hline Hipercolesterolemia & 22,9 & $21,1-24,8$ \\
\hline Diabetes & 20,6 & $19,2-22,1$ \\
\hline Depressão & 16,0 & $14,6-17,6$ \\
\hline Doença articular crônica & 14,2 & $12,7-15,8$ \\
\hline Doença cardíaca & 12,3 & $11,1-13,5$ \\
\hline Doença respiratória pulmonar crônica & 6,2 & $5,3-7,3$ \\
\hline Acidente vascular cerebral & 4,7 & $4,1-5,4$ \\
\hline \multicolumn{3}{|l|}{ Número de doenças crônicas } \\
\hline 1 & 46,9 & $44,5-49,3$ \\
\hline 2 & 27,1 & $25,5-28,8$ \\
\hline 3 & 14,6 & $13,4-15,9$ \\
\hline 4 e mais & 11,5 & $10,3-12,8$ \\
\hline \multicolumn{3}{|l|}{$\begin{array}{l}\text { Número de medicamentos em uso para as } \\
\text { doenças crônicas }\end{array}$} \\
\hline 1 & 29,5 & $28,0-31,2$ \\
\hline 2 & 26,8 & $25,2-28,5$ \\
\hline $3-4$ & 26,6 & $25,2-28,1$ \\
\hline 5 ou mais & 13,5 & $12,2-14,9$ \\
\hline
\end{tabular}

Nota: o número máximo de missings foi 266 na variável idade.

* Percentuais ajustados por pesos amostrais e por pós-estratificação segundo idade e sexo;

** Segundo a Associação Brasileira de Empresas de Pesquisa (2013) 9.

O percentual de pessoas que referiram percepção de saúde muito ruim/ruim $(9,4 \%)$ foi menor do que aquelas que referiram sua saúde como muito boa/boa ou regular. Hipertensão arterial foi a DCNT de maior prevalência na população $(69,7 \%)$, seguida da hipercolesterolemia $(22,9 \%)$, diabetes $(20,6 \%)$ e depressão (16\%). A maioria (46,9\%) relatou ter somente uma DCNT, e 11,5\% relataram quatro ou mais. Apenas 13,5\% dos entrevistados relataram estar em uso de cinco ou mais medicamentos crônicos, maiores percentuais foram encontrados com o uso de 2 a 4 medicamentos crônicos (Tabela 1).

O SUS foi a fonte de obtenção de medicamentos mais utilizada, tendo-se encontrado 39,4\% de indivíduos SUS-exclusivos, ou seja, utilizam o SUS para consultas e para obtenção dos medicamentos crônicos para sua(s) doença(s) e apenas 10,3\% se declaram usuários da farmácia popular. Também foi obtido alto percentual de indivíduos usuários do SUS que recorrem às farmácias privadas como fonte única de medicamentos $(21,8 \%)$ (Tabela 2$)$. 


\section{Tabela 2}

Uso de fontes de obtenção de medicamentos crônicos por adultos e idosos (20 anos ou mais) usuários do Sistema Únido de Saúde (SUS) com relato de doenças crônicas não transmissíveis (DCNT) e com indicação de tratamento medicamentoso segundo variáveis demográficas, socioeconômicas e de saúde. Pesquisa Nacional sobre o Acesso, Utilização e Promoção do Uso Racional de Medicamentos no Brasil (PNAUM), Brasil, 2014 (N = 6.889).

\begin{tabular}{|c|c|c|c|c|c|c|c|c|c|}
\hline \multirow[t]{2}{*}{ Variável } & \multicolumn{2}{|c|}{ SUS-exclusivos } & \multicolumn{2}{|c|}{$\begin{array}{l}\text { Usuários da farmácia } \\
\text { popular }\end{array}$} & \multicolumn{2}{|c|}{$\begin{array}{c}\text { Usuários da farmácia } \\
\text { privada }\end{array}$} & \multicolumn{2}{|c|}{$\begin{array}{l}\text { Usuários de todas as } \\
\text { fontes }\end{array}$} & \multirow[t]{2}{*}{ Valor de $p$ * } \\
\hline & $\%$ & IC95\% & $\%$ & IC95\% & $\%$ & IC95\% & $\%$ & IC95\% & \\
\hline Amostra & 39,4 & $36,4-42,4$ & 10,3 & $8,9-11,9$ & 21,8 & $19,7-24,0$ & 28,5 & $26,4-30,7$ & \\
\hline Situação conjugal & & & & & & & & & 0,339 \\
\hline Com companheiro & 38,5 & $35,2-42,9$ & 11,1 & $9,3-13,2$ & 22,1 & $19,7-24,6$ & 28,3 & $25,6-31,3$ & \\
\hline Sem companheiro & 40,5 & $36,8-44,3$ & 8,9 & $7,3-10,9$ & 21,5 & $18,5-24,8$ & 29,1 & $26,2-32,2$ & \\
\hline Cor da pele & & & & & & & & & 0,101 \\
\hline Branca & 37,5 & $34,3-40,7$ & 10,8 & $8,9-13,2$ & 21,6 & $19,1-24,5$ & 30,1 & $27,5-32,8$ & \\
\hline Não branca & 41,5 & $37,8-45,2$ & 10,0 & $8,4-11,9$ & 21,9 & $19,3-24,9$ & 26,6 & $24,1-29,2$ & \\
\hline $\begin{array}{l}\text { Idade (anos } \\
\text { completos) }\end{array}$ & & & & & & & & & $<0,001$ \\
\hline $20-39$ & 33,9 & $27,7-40,6$ & 7,8 & $4,8-12,4$ & 33,2 & $27,4-39,5$ & 25,1 & $20,4-30,6$ & \\
\hline $40-59$ & 40,6 & $37,1-44,3$ & 11,2 & $9,3-13,5$ & 20,9 & $18,3-23,6$ & 27,3 & $24,4-30,5$ & \\
\hline 60 ou mais & 40,0 & $36,6-43,5$ & 10,3 & $8,8-12,0$ & 18,7 & $16,3-21,4$ & 31,0 & $28,4-33,8$ & \\
\hline Sexo & & & & & & & & & $<0,001$ \\
\hline Masculino & 46,2 & $42,2-50,3$ & 11,8 & $9,6-14,3$ & 19,8 & $16,9-23,0$ & 22,2 & $19,5-25,3$ & \\
\hline Feminino & 35,9 & $33,0-38,9$ & 9,6 & $8,1-11,3$ & 22,8 & $20,5-25,3$ & 31,7 & $29,4-34,2$ & \\
\hline $\begin{array}{l}\text { Escolaridade (anos } \\
\text { de estudo) }\end{array}$ & & & & & & & & & 0,202 \\
\hline Não estudou & 35,6 & $31,0-40,5$ & 8,9 & $6,3-12,5$ & 25,8 & $21,7-30,3$ & 29,7 & $25,3-34,6$ & \\
\hline $1-8$ & 39,9 & $36,4-43,4$ & 11,7 & $9,8-14,0$ & 20,7 & $18,1-23,6$ & 27,7 & $25,1-30,4$ & \\
\hline $9-11$ & 40,2 & $36,4-44,1$ & 9,5 & $7,5-11,8$ & 22,0 & $19,2-25,2$ & 28,3 & $25,2-31,8$ & \\
\hline $\begin{array}{l}\text { Classificação } \\
\text { econômica ** }\end{array}$ & & & & & & & & & 0,08 \\
\hline $\mathrm{A} / \mathrm{B}$ & 34,4 & $29,5-39,6$ & 11,6 & $8,9-14,8$ & 22,3 & $18,4-26,8$ & 31,8 & $26,5-37,5$ & \\
\hline C & 40,3 & $37,1-43,7$ & 10,9 & $9,1-12,9$ & 21,4 & $19,2-23,8$ & 27,4 & $25,0-29,9$ & \\
\hline $\mathrm{D} / \mathrm{E}$ & 41,0 & $36,9-45,2$ & 7,9 & $6,0-10,4$ & 22,3 & $18,7-26,3$ & 28,8 & $25,5-32,3$ & \\
\hline $\begin{array}{l}\text { Plano de saúde ou } \\
\text { convênio médico }\end{array}$ & & & & & & & & & $<0,001$ \\
\hline Sim & 29,3 & $24,3-35,0$ & 12,9 & $9,6-17,0$ & 22,1 & $17,3-27,8$ & 35,7 & $28,7-43,4$ & \\
\hline Não & 40,6 & $37,6-43,7$ & 10,0 & $8,6-11,7$ & 21,7 & $19,5-24,1$ & 27,7 & $25,6-29,8$ & \\
\hline $\begin{array}{l}\text { Autopercepção da } \\
\text { saúde }\end{array}$ & & & & & & & & & $<0,001$ \\
\hline Muito bom/Bom & 44,4 & $40,6-48,2$ & 12,1 & $10,0-14,6$ & 20,5 & $17,8-23,4$ & 23,0 & $20,7-25,6$ & \\
\hline Regular & 36,7 & $33,2-40,2$ & 9,2 & $7,6-11,1$ & 22,3 & $19,7-25,2$ & 31,8 & $28,6-35,1$ & \\
\hline Muito ruim/Ruim & 27,8 & $22,8-33,4$ & 6,7 & $4,8-9,2$ & 25,3 & $20,8-30,5$ & 40,2 & $35,1-45,6$ & \\
\hline Hipertensão arterial & 41,3 & $37,9-44,7$ & 12,0 & $10,4-13,8$ & 17,3 & $15,1-19,7$ & 29,5 & $27,1-31,9$ & $<0,001$ \\
\hline Hipercolesterolemia & 34,1 & $30,2-38,2$ & 11,5 & $9,2-14,2$ & 18,6 & $15,3-22,5$ & 35,8 & $32,0-39,8$ & $<0,001$ \\
\hline Diabetes & 42,1 & $38,2-46,0$ & 10,1 & $8,0-12,6$ & 13,2 & $10,7-16,1$ & 34,7 & $31,3-38,2$ & $<0,001$ \\
\hline Depressão & 34,7 & $30,4-39,4$ & 8,2 & $6,0-11,2$ & 19,4 & $15,3-24,4$ & 37,6 & $33,1-42,4$ & $<0,001$ \\
\hline $\begin{array}{l}\text { Doença articular } \\
\text { crônica }\end{array}$ & 23,0 & $19,0-27,6$ & 9,5 & $7,2-12,6$ & 25,9 & $21,9-30,5$ & 41,5 & $37,2-45,9$ & $<0,001$ \\
\hline Doença cardíaca & 31,8 & $26,8-37,4$ & 12,0 & $9,0-15,9$ & 21,1 & $17,3-25,5$ & 35 & $30,5-39,8$ & $<0,001$ \\
\hline $\begin{array}{l}\text { Doença respiratória } \\
\text { pulmonar crônica }\end{array}$ & 27,4 & $21,6-34,1$ & 6,2 & $3,9-9,6$ & 24,3 & $18,2-31,6$ & 42,1 & $35,3-49,4$ & $<0,001$ \\
\hline
\end{tabular}

(continua) 
Tabela 2 (continuação)

\begin{tabular}{|c|c|c|c|c|c|c|c|c|c|}
\hline \multirow[t]{2}{*}{ Variável } & \multicolumn{2}{|c|}{ SUS-exclusivos } & \multicolumn{2}{|c|}{$\begin{array}{c}\text { Usuários da farmácia } \\
\text { popular }\end{array}$} & \multicolumn{2}{|c|}{$\begin{array}{c}\text { Usuários da farmácia } \\
\text { privada }\end{array}$} & \multicolumn{2}{|c|}{$\begin{array}{c}\text { Usuários de todas as } \\
\text { fontes }\end{array}$} & \multirow[t]{2}{*}{ Valor de $p$ * } \\
\hline & $\%$ & IC95\% & $\%$ & IC95\% & $\%$ & IC95\% & $\%$ & IC95\% & \\
\hline $\begin{array}{l}\text { Acidente vascular } \\
\text { cerebral }\end{array}$ & 32,9 & $26,8-39,8$ & 11,3 & $7,6-16,6$ & 18,5 & $13,5-24,8$ & 37,3 & $30,7-44,4$ & 0,035 \\
\hline $\begin{array}{l}\text { Número de } \\
\text { medicamentos para } \\
\text { as doenças crônicas }\end{array}$ & & & & & & & & & $<0,001$ \\
\hline 1 & 43,8 & $39,3-48,5$ & 10,9 & $8,4-13,9$ & 29,6 & $26,1-33,4$ & 15,7 & $13,3-18,5$ & \\
\hline 2 & 42,4 & $38,2-46,8$ & 12,2 & $9,8-15,0$ & 20,5 & $17,8-23,4$ & 25,0 & $21,9-28,3$ & \\
\hline $3-4$ & 37,7 & $34,2-41,4$ & 9,3 & $7,7-11,3$ & 16,3 & $13,7-19,3$ & 36,6 & $33,4-39,9$ & \\
\hline 5 ou mais & 30,0 & $24,7-36,0$ & 8,5 & $6,2-11,6$ & 12,5 & $8,4-18,1$ & 49,0 & $43,0-54,9$ & \\
\hline
\end{tabular}

Nota: o número máximo de missings foi 266 na variável idade.

* Teste qui-quadrado de Pearson;

** Segundo a Associação Brasileira de Empresas de Pesquisa (2013) 9.

Não foram encontradas diferenças entre situação conjugal, cor da pele, escolaridade ou classe econômica. Entre as faixas etárias estudadas, aqueles com 40 anos ou mais foram os que mais se mostraram SUS-exclusivos, bem como aqueles que declararam não possuir plano de saúde e os que perceberam sua saúde como boa ou muito boa. Entre os usuários SUS-exclusivos, os homens foram a maioria, já entre os usuários de todas as fontes, a maioria foi de mulheres. Nas demais fontes, não há diferença estatística entre os sexos (Tabela 2).

Na população estudada, os indivíduos das classes econômicas A e B são os que mais utilizam todas as fontes de obtenção de medicamentos $(31,8 \%)$ e os indivíduos das classes D e E são os que mais utilizam exclusivamente o SUS (41\%) (Tabela 2).

Quando há o uso de 1 e de 2 medicamentos para as doenças crônicas, o percentual de uso da fonte de obtenção SUS-exclusivos, respectivamente 43,8\% e 42,4\%, é superior aos percentuais obtidos para usuários da farmácia popular (10,9\% e 12,2\%), usuários da farmácia privada (29,6\% e 20,5\%) e usuários de todas as fontes de obtenção (15,7\% e 25\%). Somente quando há o uso de 5 ou mais medicamentos para as doenças crônicas, o percentual de uso de todas as fontes de obtenção (49\%) supera o uso SUS -exclusivo (30\%) (Tabela 2).

O maior percentual de utilização da fonte de obtenção SUS-exclusivo foi entre os portadores de hipertensão arterial $(41,3 \%)$ e de diabetes $(42,1 \%)$. Portadores de doença articular crônica tiveram o menor percentual (23\%) de utilização dessa fonte de obtenção (Tabela 2).

A proporção de indivíduos SUS-exclusivos diminuiu para o aumento do número de doenças crônicas. Quando o indivíduo relatou ter uma ou duas doenças crônicas, observou-se que os maiores percentuais de uso de fontes de obtenção de medicamentos se referem aos SUS-exclusivos (respectivamente 46,6\% e 37,9\%). Quando o número de doenças crônicas é 3 ou 4, os maiores percentuais de uso de fontes de obtenção se referem à categoria todas as fontes de obtenção (respectivamente $41 \% \mathrm{e}$ 48,2\%) (Figura 1).

Os portadores de diabetes e de hipertensão arterial são os que mais utilizam o SUS como fonte exclusiva de obtenção de seus medicamentos. Os portadores de doença respiratória pulmonar crônica e de doença articular crônica são os indivíduos que mais utilizaram a farmácia privada e que buscaram medicamentos em todas as fontes de obtenção (Figura 2).

Na média do país, o SUS foi a principal fonte de obtenção de medicamentos de uso crônico. Em todo o Brasil, a utilização da farmácia popular teve a menor expressão (Figura 3).

No Norte, Nordeste e Centro-oeste, não houve diferença estatística entre a utilização exclusiva do SUS e a utilização da farmácia privada como fonte de obtenção de medicamentos crônicos. Já no Sudeste e no Sul, houve maior utilização do SUS em relação à farmácia privada (Figura 3). 


\section{Figura 1}

Uso de fontes de obtenção de medicamentos crônicos por adultos e idosos (20 anos ou mais) usuários do Sistema Único de Saúde (SUS) com doenças crônicas não transmissíveis (DCNT) e com indicação de tratamento medicamentoso segundo o número de DCNT. Pesquisa Nacional sobre o Acesso, Utilização e Promoção do Uso Racional de Medicamentos no Brasil (PNAUM), Brasil, 2014.

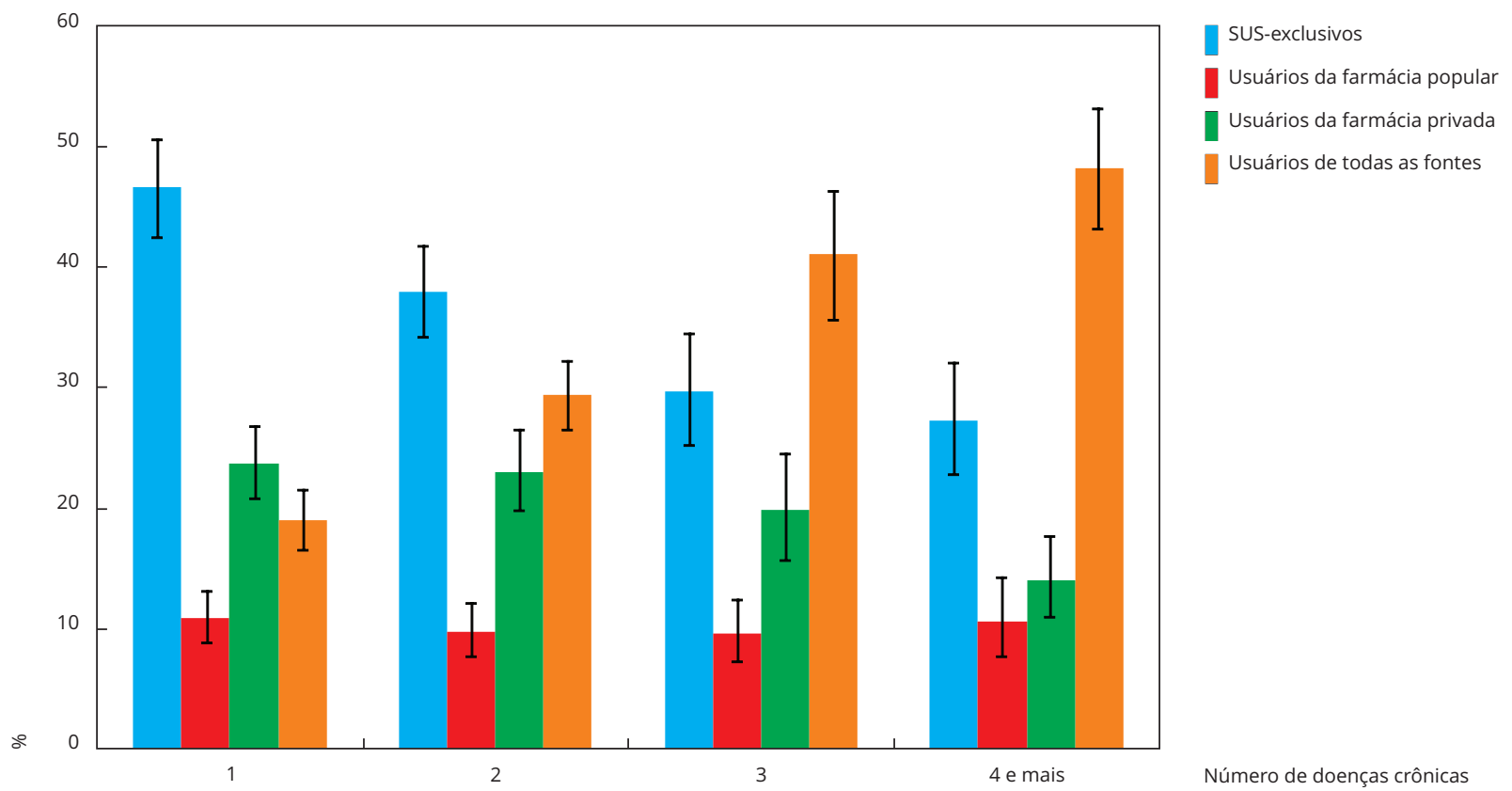

\section{Discussão}

A população deste estudo, formada por portadores de DCNT com indicação de tratamento medicamentoso e que são usuários do SUS, possui média de idade de 59 anos e níveis de renda e de escolaridade baixos. São indivíduos que utilizam o sistema público para o atendimento médico, uma vez que $89,7 \%$ não possuíam plano de saúde ou convênio médico. Na análise das Pesquisas de Orçamentos Familiares (POF) de 2002-2003 e 2008-2009, verificou-se que o gasto com planos de saúde está concentrado entre as famílias com maior renda 10.

A proporção de indivíduos SUS-exclusivos, isto é, que receberam prescrição no SUS e obtiveram seus medicamentos crônicos no sistema público de saúde, correspondeu a menos da metade da população estudada, resultado semelhante ao encontrado por Boing et al. ${ }^{11}$ quando analisaram dados oriundos do suplemento saúde da Pesquisa Nacional por Amostra de Domicílios (PNAD) conduzida em 2008. Ainda que prevaleça a obtenção de medicamentos para doenças crônicas nas farmácias do SUS, houve expressiva proporção de usuários do SUS que adquiriram medicamentos em todas as fontes de obtenção, ou que conseguiram seus medicamentos por meio da compra em farmácias privadas. A utilização do setor privado e de copagamento por usuários do SUS para garantir o acesso a medicamentos implica maior comprometimento da renda nos gastos em saúde, o que penaliza, sobretudo, os mais pobres 11 .

Algumas dificuldades para o acesso a medicamentos no setor público podem motivar a população que recebe a prescrição no SUS a procurar outras fontes para obter os medicamentos para tratamento de doenças crônicas, seja custeando integralmente o tratamento medicamentoso, seja utilizando o copagamento para alguns itens do PFPB em sua rede própria ou na sua vertente Aqui Tem Farmácia Popular. A dificuldade para encontrar todos os medicamentos que foram prescritos nas farmácias do 
Figura 2

Distribuição de fontes de obtenção de medicamentos crônicos por adultos e idosos (20 anos ou mais) usuários do Sistema Único de Saúde (SUS) com doenças crônicas não transmissíveis (DCNT) e com indicação de tratamento medicamentoso segundo doença crônica. Pesquisa Nacional sobre o Acesso, Utilização e Promoção do Uso Racional de Medicamentos no Brasil (PNAUM), Brasil, 2014.

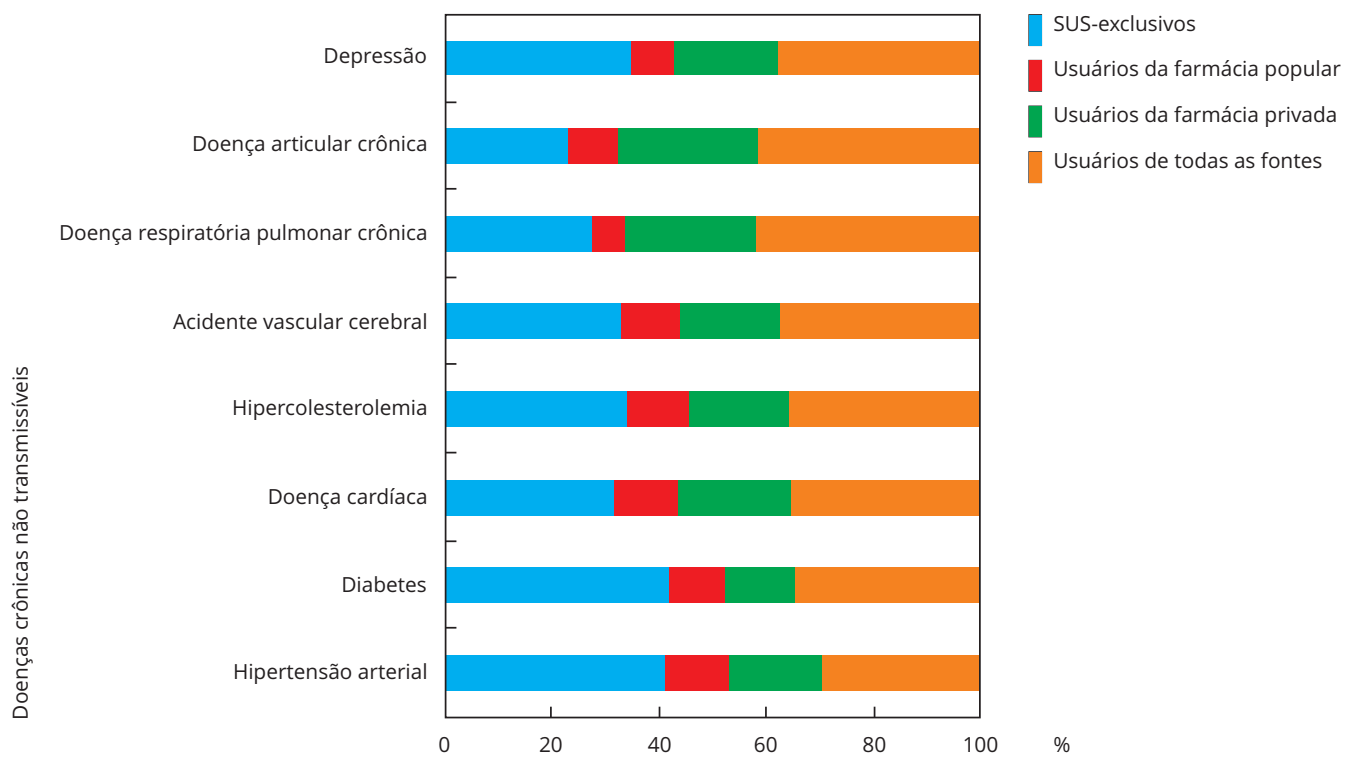

SUS é uma delas. Baixa proporção de unidades básica de saúde (UBS) com disponibilidade total dos medicamentos-chave, os quais incluíram medicamentos de uso crônico como os anti-hipertensivos, antidiabéticos e cardiológicos, foi encontrada no estudo de Mendes et al. 4 realizado nas UBS do Brasil em 2014. Outras dificuldades podem estar relacionadas às questões de capacidade aquisitiva, acessibilidade geográfica e aceitabilidade, que formam, junto com a disponibilidade física, as quatro dimensões do modelo teórico sobre o acesso proposto por Penchansky \& Thomas 12 e adaptado por Luiza \& Bermudez 13.

A idade e o sexo são as variáveis demográficas que influenciam o tipo de fonte de obtenção de medicamentos utilizada. Observou-se que a proporção de usuários da farmácia privada diminuiu com o aumento da idade, enquanto a proporção de usuários da farmácia popular aumentou com a idade.

A necessidade de polifarmácia, situação comum de se estabelecer com o aumento da idade, propicia aumento nos gastos com medicamentos, estimulando sua busca na farmácia popular onde os preços são menores. Costa et al. ${ }^{14}$, em estudo de base populacional no Município de Campinas (São Paulo) em 2008, encontraram maior utilização do PFPB entre as mulheres e indivíduos acima de 70 anos de idade.

Entre os usuários de todas as fontes de obtenção, as mulheres sobressaíram em relação aos homens. É possível que as mulheres tendam a percorrer várias fontes e a terem mais prontidão para desembolsar para ter acesso aos medicamentos. Tal comportamento pode ser atribuído à maior percepção da mulher quanto aos sintomas e sinais físicos das doenças e do conhecimento adquirido no desempenho do seu papel social de cuidadora da família 15.

Indivíduos de todas as classes econômicas se mostraram usuários da farmácia privada, pois os percentuais de utilização de todas as fontes de obtenção foram próximos. A provisão de medicamentos nas farmácias do SUS atende predominantemente aos menos favorecidos, pois entre os indivíduos SUS-exclusivos, a maior parte pertence às classes econômicas $\mathrm{C}, \mathrm{D}, \mathrm{E}$. Esses resultados refletem a 
Figura 3

Uso das fontes de obtenção de medicamentos crônicos por adultos e idosos (20 anos ou mais) usuários do Sistema Único de Saúde (SUS) com doenças crônicas não transmissíveis (DCNT) e com indicação de tratamento medicamentoso segundo região geográfica de residência. Pesquisa Nacional sobre o Acesso, Utilização e Promoção do Uso Racional de Medicamentos no Brasil (PNAUM), Brasil, 2014.

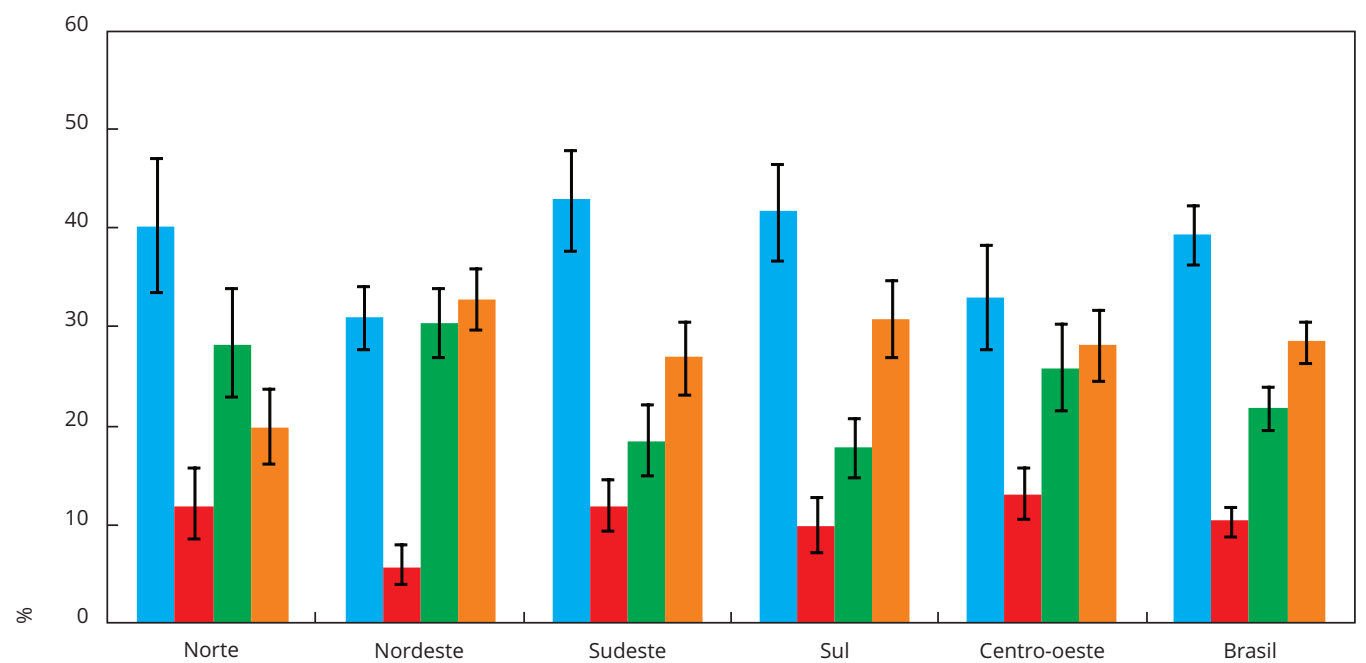

$\Pi$ SUS-exclusivos

Usuários da farmácia popular

Usuários da farmácia privada

Usuários de todas as fontes

Sul

Centro-oeste

Brasil

relevância da provisão de medicamentos pelo SUS a fim de promover equidade no acesso aos medicamentos 11,16. Como a maioria da população que utiliza exclusivamente o SUS como fonte de obtenção pertence às classes econômicas mais baixas, é possível que os indivíduos SUS-exclusivos, que possuem plano de saúde, recebam ajuda de seus familiares ou comprometam grande parte do orçamento familiar para esse custeio 10 .

$\mathrm{Na}$ população estudada, as maiores prevalências autorreferidas de doenças crônicas foram para hipertensão arterial, diabetes e hipercolesterolemia, doenças crônicas associadas com o desenvolvimento de problemas cardiovasculares, e são, juntamente com distúrbios neuropsiquiátricos, as principais causas da carga de doenças no Brasil 1.

Os indivíduos que utilizam exclusivamente o SUS como fonte de obtenção de medicamentos sofrem, majoritariamente, de apenas uma doença crônica e utilizam um medicamento crônico. Essa proporção decai à medida que os indivíduos apresentam mais doenças crônicas e utilizam mais medicamentos. Pode-se interpretar que aumenta a possibilidade de não obtenção do tratamento completo no SUS quando ele envolve muitos medicamentos 4,17. A mesma tendência de queda é encontrada entre aqueles que obtêm medicamentos em farmácias privadas à medida que aumenta o número de DCNT e de medicamentos, porém, neste caso, pode-se atribuir ao aumento dos gastos quando o tratamento é múltiplo. A proporção de usuários da farmácia popular foi a menor entre os grupos de fonte de obtenção de medicamentos, mantendo-se próxima a 10\% independentemente do número de DCNT. Não obstante a baixa prevalência de utilização, o programa pode exercer papel fundamental no acesso a medicamentos crônicos de salvaguarda das falhas na provisão pública de medicamentos no país, ainda que ela se faça restrita a um elenco de medicamentos específico, com variedade menor de fármacos em relação às farmácias do SUS e privadas 3,5.

A autoavaliação do estado de saúde afeta a busca por diferentes fontes de obtenção de medicamentos. O uso de medicamentos é um fator associado à percepção ruim da saúde 18 . Naturalmente, as pessoas que utilizam muitos medicamentos têm percepção negativa do seu estado de saúde e, dessa forma, tendem a se esforçar mais para obter o tratamento completo e utilizar todas as fontes de obtenção de medicamentos. Já, quando a autoavaliação da saúde é positiva, talvez por necessitarem 
de menos medicamentos, consigam obter em apenas um tipo de fonte, por isso são a maioria entre os SUS-exclusivos e entre os usuários da farmácia popular.

Entre os usuários da farmácia popular, o Nordeste apresentou menor proporção que a Região Centro-oeste do país. Emmerick et al. 6 corroboram esses resultados porque a Região Centro-oeste apresentou os maiores índices de crescimento na cobertura da modalidade Aqui tem Farmácia Popular durante os anos de 2006 até 2012, enquanto a cobertura dessa modalidade do programa esteve abaixo de 50\% de todos os municípios da Região Nordeste, em 2012.

No Nordeste, não houve diferença estatística entre a utilização exclusiva do SUS e os usuários da farmácia privada, indicando que a população utiliza da mesma forma as fontes de obtenção de medicamentos. É possível que deficiências na provisão de medicamentos pelo SUS sejam frequentes e a população tenha que comprar nas farmácias privadas na vigência de problemas de disponibilidade de medicamento no SUS. Boing et al. 11, em relação à obtenção de medicamentos para hipertensão e diabetes em drogarias e/ou farmácias, encontraram maiores percentuais nas regiões Nordeste e Norte. Destaca-se que, no presente estudo, a Região Norte apresentou elevado percentual de utilização da farmácia privada pelos usuários do SUS com relato de DCNT e prescrição de medicamentos. Esses resultados refletem que a população do Nordeste e Norte do país é sacrificada no que tange ao acesso a medicamentos.

No Sudeste e no Sul, a utilização exclusiva do SUS como fonte de obtenção foi superior à utilização da farmácia privada. Similarmente, Boing et al. 11 constataram que o Sul e o Sudeste apresentaram, respectivamente, as maiores prevalências de acesso aos medicamentos no setor público entre pessoas que tiveram prescrição no próprio sistema público. Costa et al. 19 também observaram que, entre diabéticos e hipertensos, os maiores percentuais de obtenção de medicamentos em unidades de saúde foram nas regiões Sul e Sudeste do país, respectivamente.

Possíveis limitações podem ser consideradas tais como a qualidade da informação autorreferida no estudo fonte quanto à correta identificação dos locais de obtenção de medicamentos. Além do programa Aqui tem Farmácia Popular, a população pode obter medicamentos com desconto por meio de programas de fidelização a laboratórios na mesma drogaria onde há o Aqui Tem Farmácia Popular; várias farmácias no país adotam nome fantasia que faz lembrar o programa governamental PFPB. E quanto ao fornecimento dos medicamentos financiados pelo SUS, pode ocorrer por meio de sistemas mistos, em que parte dos medicamentos está disponível nas farmácias dos serviços de saúde e parte na farmácia centralizada.

\section{Conclusão}

Este estudo foi realizado com dados de uma grande pesquisa nacional e aborda questões importantes sobre a realidade da assistência farmacêutica no SUS, especialmente sobre as fontes formais para obtenção de medicamentos de que a população dispõe atualmente.

Os usuários do SUS têm como sua fonte primária de medicamentos o próprio SUS, todavia há uma participação relevante de "todas as fontes", indicando problemas no acesso dentro do SUS.

No Sul e Sudeste, a população busca menos a farmácia privada do que no Nordeste e Norte do país para obter medicamentos de uso crônico, possivelmente pelas deficiências na provisão de tais medicamentos pelo SUS nessas regiões.

Sugere-se que estudos futuros identifiquem os medicamentos mais procurados em cada fonte de obtenção e também testem a influência de determinantes do acesso a medicamentos na escolha da fonte de obtenção de medicamentos pela população, a fim de orientar intervenções prioritárias por parte do poder público. 


\section{Colaboradores}

S. R. Matta e A. D. Bertoldi participaram da concepção, execução das análises, redação e aprovação da versão final do manuscrito. I. C. M. Emmerick e V. L. Luiza participaram da concepção, redação e aprovação final do manuscrito. A. T. Fontanella e K. S. Costa participaram da revisão crítica do conteúdo intelectual, redação e aprovação final do manuscrito.

\section{Outros membros do Grupo PNAUM}

L. R. Ramos, M. R. Farias, M. A. Oliveira, N. U. L. Tavares, P. S. D. Arrais, S. S. Mengue, T. S. Dal Pizzol.

\section{Agradecimentos}

Ao Ministério da Saúde por meio da Secretaria de Ciência e Tecnologia e Insumos Estratégicos (SCTIE/MS) pelo financiamento e apoio técnico para a realização da Pesquisa Nacional sobre Acesso, Utilização e Promoção do Uso Racional de Medicamentos e, em especial, à equipe de suporte do projeto, nos nomes de Amanda Ramalho da Silva e Luciano Santos Pinto Guimarães.

\section{Referências}

1. Schmidt MI, Duncan BB, Azevedo e Silva G, Menezes AM, Monteiro CA, Barreto SM, et al. Chronic non-communicable diseases in Brazil: burden and current challenges. Lancet 2011; 377:1949-61.

2. Theme Filha MM, Souza Junior PRB, Damacena GN, Szwarcwald CL. Prevalência de doenças crônicas não transmissíveis e associação com autoavaliação de saúde: Pesquisa Nacional de Saúde, 2013. Rev Bras Epidemiol 2015; 18: 83-96.

3. Oliveira MA, Luiza VL, Tavares NUL, Mengue SS, Arrais PSD, Farias MR, et al. Access to medicines for chronic diseases in Brazil: a multidimensional approach. Rev Saúde Pública 2016; 50 Suppl 2:6s.

4. Mendes LV, Campos MR, Chaves GC, Silva RM, Freitas PS, Costa KS, et al. Disponibilidade de medicamentos nas unidades básicas de saúde e fatores relacionados: uma abordagem transversal. Saúde Debate 2014; 38(n.spe):109-23.

5. Silva RM, Caetano R. Programa "Farmácia Popular do Brasil”: caracterização e evolução entre 2004-2012. Ciênc Saúde Coletiva 2015; 20:2943-56.

6. Emmerick ICM, do Nascimento Jr. JM, Pereira MA, Luiza VL, Ross-Degnan D; ISAUM-Br Collaborative Group. Farmácia Popular Program: changes in geographic accessibility of medicines during ten years of a medicine subsidy policy in Brazil. J Pharm Policy Pract 2015; 8:10.

7. Luiza VL, Chaves LA, Silva RM, Emmerick ICM, Chaves GC, Araújo SCF, et al. Pharmaceutical policies: effects of cap and co-payment on rational use of medicines. Cochrane Database Syst Rev 2015; (5):CD007017.
8. Mengue SS, Bertoldi AD, Boing AC, Tavares NUL, Dal Pizzol TS, Oliveira MA, et al. National Survey on Access, Use and Promotion of Rational Use of Medicines (PNAUM): household survey component methods. Rev Saúde Pública 2016; 50 Suppl 2:4s.

9. Associação Brasileira de Empresas de Pesquisa. Critério de Classificação Econômica Brasil. http://www.abep.org.br (acessado em 07/ Dez/2016).

10. Garcia LP, Ocké-Reis CO, Magalhães LCG, Sant'Anna AC, Freitas LRS. Gastos com planos de saúde das famílias brasileiras: estudo descritivo com dados das Pesquisas de Orçamentos Familiares 2002-2003 e 2008-2009. Ciênc Saúde Coletiva 2015; 20:1425-34.

11. Boing AC, Bertoldi AD, Boing AF, Bastos JL, Peres KG. Access to medicines in the public sector: analysis of users of the Brazilian Unified National Health System. Cad Saúde Pública 2013; 29:691-701.

12. Penchansky R, Thomas JW. The concept of access: definition and relationship to consumer satisfaction. Med Care 1981; 19:127-40.

13. Luiza VL, Bermudez JAZ. Acesso a medicamentos: conceitos e polêmicas. In: Bermudez JAZ, Oliveira MA, Esher A, organizadores. Acesso a medicamentos: derecho fundamental, papel del Estado. Rio de Janeiro: Escola $\mathrm{Na}$ cional de Saúde Pública, Fundação Oswaldo Cruz; 2004. p. 45-67.

14. Costa KS, Francisco PMSB, Barros MBA. Conhecimento e utilização do Programa Farmácia Popular do Brasil: estudo de base populacional no município de Campinas-SP. Epidemiol Serv Saúde 2014; 23:397-408. 
15. Barros MBA, Francisco PMSB, Zanchetta LM, César CLG. Tendências das desigualdades sociais e demográficas na prevalência de doenças crônicas no Brasil, PNAD: 2003-2008. Ciênc Saúde Coletiva 2011; 16:3755-68.

16. Costa KS, Tavares NUL, Mengue SS, Pereira MA, Malta DC, Silva Júnior JB. Obtenção de medicamentos para hipertensão e diabetes no Programa Farmácia Popular do Brasil: resultados da Pesquisa Nacional de Saúde, 2013. Epidemiol Serv Saúde 2016; 25:22-44.

17. Pinto CDBS, Miranda ES, Emmerick ICM, Costa NR, Castro CGSO. Preços e disponibilidade de medicamentos no Programa Farmácia Popular do Brasil. Rev Saúde Pública 2010; 44:611-9.

\section{Abstract}

The article aimed to identify the sources of medicines for users of the Brazilian Unified National Health System (SUS) that reported chronic noncommunicable diseases, characterizing the group according to demographic, socioeconomic, and health variables in the different regions of Brazil. Data were analyzed from the National Survey on Access, Use, and Promotion of Rational Use of Medicines in Brazil (PNAUM), a crosssectional population-based study. The dependent variable was "source of medicines", and the associations were analyzed with demographic, socioeconomic, and health status variables. Users that obtained care and medicines solely in the SUS were designated here as SUS-exclusive. Some 39\% of persons obtained their medicines from SUS pharmacies and 28.5\% from other sources. The study found $42.9 \%, 41.8 \%, 40.2 \%$, and $31 \%$ of exclusive SUS users in the Southeast, South, North, and Northeast regions of Brazil, respectively. SUS users have the SUS as their primary source of medicines, but there is also a relevant share from other sources, suggesting problems with access in the SUS. In the South and Southeast regions, the population relies less on private pharmacies than in the Northeast and North.

Health Services Accessibility; Unified Health System; Chronic Disease; Pharmaceutical Preparations
18. Pagotto V, Nakatani AYK, Silveira EA. Fatores associados à autoavaliação de saúde ruim em idosos usuários do Sistema Único de Saúde. Cad Saúde Pública 2011; 27:1593-602.

19. Costa KS, Francisco PMSB, Malta DC, Barros MBA. Fontes de obtenção de medicamentos para hipertensão e diabetes no Brasil: resultados de inquérito telefônico nas capitais brasileiras e no Distrito Federal, 2011. Cad Saúde Pública 2016; 32:e0009014.

\section{Resumen}

El objetivo de este artículo fue identificar las fuentes de obtención de medicamentos, utilizados por usuarios del Sistema Único de Salud brasileño (SUS), con antecedentes de enfermedades crónicas, caracterizando tanto a la población, como las variables demográficas, socioeconómicas y de salud, en las diferentes regiones del país. Se analizaron los datos de la Encuesta Nacional sobre el Acceso, Utilización y Promoción del Uso Racional de Medicamentos en Brasil (PNAUM), un estudio transversal de base poblacional. La variable dependiente fue la 'fuente de obtención de medicamentos' $y$ se analizaron asociaciones con variables demográficas, socioeconómicas y con el estado de salud. Los usuarios que recibieron cuidados y medicamentos únicamente en el SUS fueron designados aquí como SUS exclusivos. Cerca de un $39 \%$ de las personas obtuvieron sus medicamentos exclusivamente en farmacias del SUS y un 28,5\% recurrieron a otras fuentes. Se encontraron un $42,9 \%, 41,8 \%, 40,2 \%, 31 \%$, usuarios SUS exclusivos, respectivamente, en el Sudeste, Sur, Norte, y Nordeste. Los usuarios del SUS tienen como fuente primaria de medicamentos el propio SUS, no obstante, existe una participación relevante de otras fuentes de obtención, indicando problemas en el acceso dentro del SUS. En el Sur y Sudeste, la población busca menos la farmacia privada que en el Nordeste y Norte del país.

Accesibilidad a los Servicios de Salud; Sistema Unico de Salud; Enfermedad Crónica;

Preparaciones Farmacéuticas
Recebido em 04/Mai/2017

Versão final reapresentada em 06/Set/2017

Aprovado em 15/Set/2017 